\title{
Endoscopic Therapy of Early Carcinoma of the Oesophagus
}

\author{
Mate Knabe Andrea May Christian Ell \\ Department of Medicine II/IV, Sana Klinikum Offenbach, Offenbach, Germany
}

\section{Keywords}

Barrett - Early oesophageal cancer .

Endoscopic resection - Squamous cell carcinoma . Oesophageal adenocarcinoma

\section{Summary}

Background: Oesophageal cancer is a comparatively rare disease in the Western world. Prognosis is highly dependent on the choice of treatment. Early stages can be treated by endoscopic resection, whereas surgery needs to be performed in the case of advanced carcinomas. Technical progress has enabled high-definition endoscopes and technical add-ons which help the endoscopist in finding fine irregularities in the oesophageal mucosa, though interpretation still remains challenging. Methods: In this review, we discuss both novel and old diagnostic procedures and their value, as well as the current recommendations for the diagnosis and treatment of early oesophageal carcinomas. The database of PubMed and Medline was searched and analysed to provide all relevant literature for this review. Results and Conclusion: Endoscopic resection is the therapy of choice in early oesophageal cancer. In case of adenocarcinoma it is mandatory to perform subsequent ablation of all residual Barrett's mucosa to avoid metachronous lesions.

(c) 2015 S. Karger GmbH, Freiburg

\section{Introduction}

Due to the absence of symptoms, most patients who present with oesophageal cancer have advanced disease at the time of the initial diagnosis. Patients who show symptoms and probably are also at familial risk should therefore undergo an upper gastrointestinal endoscopy as soon as possible. An examination in time may make it possible to diagnose the cancer at an earlier stage and offers an option for curative treatment. Although endoscopic examination in patients at risk (obesity, gastro-oesophageal reflux disease, age $>50$ years, Caucasian, hiatal hernia) is recommended, general screening endoscopy is discussed controversially [1]. Thus far, there have been no prospective randomized trials proving an advantage of screening programs for patients with reflux symptoms in order to decrease the incidence or the mortality rate. $80 \%$ of patients diagnosed with oesophageal cancer have no reflux symptoms [2]. Advanced imaging techniques and high-definition video endoscopes have recently entered clinical practice and can help examiners to identify diminutive lesions in the oesophagus. However, interpreting the findings and drawing the right conclusions will continue to be a challenge for the examiner.

Oesophageal cancer is a comparatively rare disease in the Western world. It represents only $1 \%$ of all malignancies in Europe. The current incidence varies among regions worldwide - from 3-6 per 100,000 in Germany to 25 per 100,000 in Asia [3]. In 2012, 465,000 cases were diagnosed, with a high rate of mortality of up to 400,000 in the same year $[3,4]$. Adenocarcinoma (AC) is generally found more often in highly developed countries of the Western world, whereas squamous cell carcinoma (SCC) has a higher incidence in Asia. The global incidence of oesophageal AC is 0.7 per 100,000, with a high level of variation between different countries [4]. The global incidence for oesophageal SCC is 5.2 per 100,000 [4]. The different incidence is highly dependent on the varying risk factors for each cancer type. Known risk factors include male sex and an increased familial incidence of Barrett's oesophagus and Barrett's AC [5]. Furthermore, there seems to be an association between Barrett's AC and obesity [6]. Nicotine and alcohol abuse play a role in the pathogenesis of SCC [7]. Opinions vary regarding the lifetime risk of AC developing in patients known to have Barrett's oesophagus; however, an annual carcinoma incidence of $0.12-0.5 \%$ may be assumed [8-10].

\section{KARGER \\ Fax +497614520714

\section{(c) 2015 S. Karger GmbH, Freiburg}

1662-6664/15/0315-0320\$39.50/0
Dr. Mate Knabe

Medizinische Klinik II/IV - Gastroenterologie, Pneumologie, Hämatologie-Onkologie Sana Klinikum Offenbach GmbH

Starkenburgring 66, 63069 Offenbach, Germany

mate.knabe@sana.de 


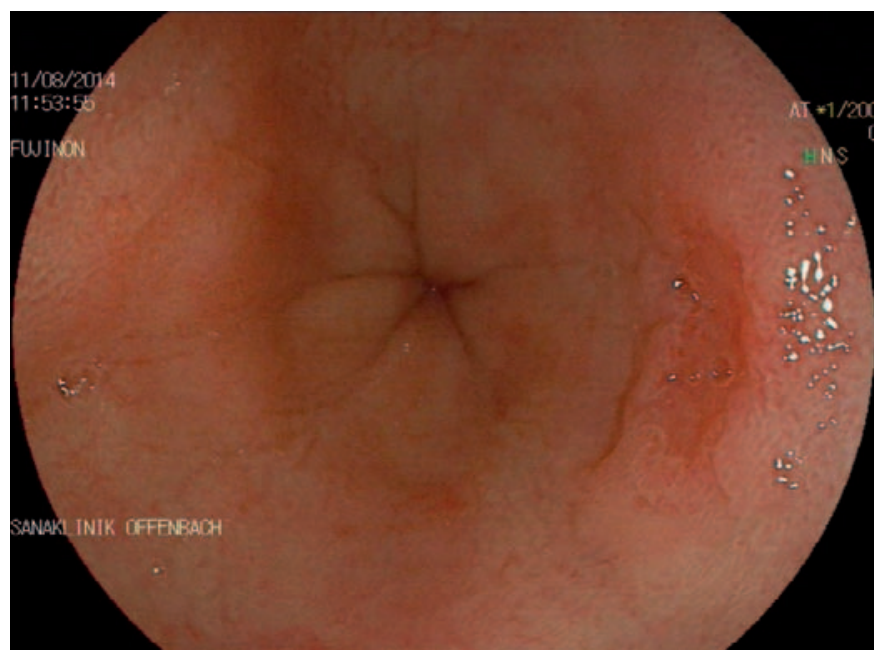

Fig. 1. Upper gastrointestinal endoscopy of the oesophagus in a patient suffering from reflux. At the 3 o'clock position there is a small type IIc lesion highly suspicious for early adenocarcinoma.

Oesophageal cancer should be histologically differentiated into SCC and AC; all other types are extremely rare. SCCs are mainly found in the upper and middle oesophagus, while AC is mainly found in the lower part. Both entities develop from low-grade intraepithelial neoplasia (LGIN) via high-grade intraepithelial neoplasia (HGIN) to invasive cancer. AC develops from a form of intestinal metaplasia known as Barrett's mucosa, which is considered to be a premalignant condition. The term 'adenocarcinoma of the oesophagogastric junction' (AEJ) encompasses several different tumour entities: Barrett's carcinoma (AEJ I), which develops from intestinal metaplasia; carcinoma of the cardia (AEJ II); and subcardial cancer (AEJ III). However, the endoscopic treatment for these different types of lesion is the same.

\section{Endoscopic Diagnosis}

Detecting neoplastic lesions in the oesophagus can be challenging even for experienced endoscopists. Early diagnosis is essential in order to allow endoscopic therapy, which is associated with an excellent prognosis. High-definition endoscopes have a good sensitivity for diagnosing early neoplasia. Modern video chips provide an extremely high resolution ( $>1,000,000$ pixels) and can display the mucosa and the different structures of neoplastic and non-neoplastic lesions. However, longer inspection times and greater experience on the part of the examiner are strongly associated with increased detection rates for dysplasia [11]. Barrett's metaplasia, a precancerous condition, has a reddish appearance during upper gastrointestinal endoscopy. A villous structure is the typical appearance during endoscopy (fig. 1). The full extent of all Barrett's epithelium should be described using the Prague classification [12, 13]. This includes a description of the full circumferential length $(\mathrm{C})$ and the maximum length $(\mathrm{M})$ in centimetres. The maximum length is used to differentiate between long-segment $(\geq 3 \mathrm{~cm})$ and

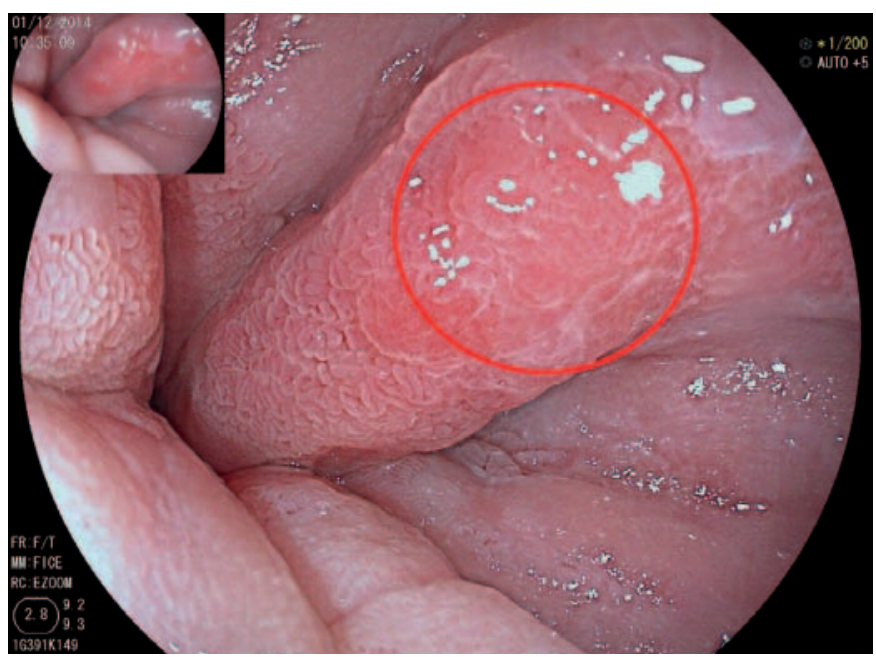

Fig. 2. Another patient with Barrett's oesophagus after chromoendoscopy with $1.5 \%$ acetic acid. The neoplastic lesion is now clearly visible in between the Barrett's mucosa.

short-segment $(<3 \mathrm{~cm})$ Barrett's oesophagus. A detailed description of the intestinal metaplasia and suspicious areas it contains is important for purposes of surveillance, prognostic assessment, and subsequent endoscopic treatment. In addition to targeted biopsies of suspicious lesions, all patients with a first diagnosis of Barrett's oesophagus should undergo random four-quadrant biopsies every $2 \mathrm{~cm}$ (using the Seattle protocol). In case of SCC the examiner should watch out for focal areas of irregular mucosa or plaque-like lesions.

\section{Advanced Endoscopic Imaging}

Chromoendoscopy is a procedure for dyeing the gastrointestinal mucosa in order to highlight its surface structures. Application of $1.5-2.5 \%$ acetic acid leads to a temporary change in the surface proteins which reveals a typical pit pattern in Barrett's mucosa (fig. 2). During endoscopy, the examiner looks for inhomogeneities and early disappearance of the staining [11]. An alternative dye for chromoendoscopic staining is methylene blue which selectively highlights the columnar epithelium in Barrett's oesophagus. As the available data are controversial, targeted biopsies after methylene blue staining in Barrett's oesophagus should only be performed in specialized centres with a high level of expertise in this field [14, 15].

The development of high-definition video endoscopes has led to new imaging techniques. In an attempt to overcome the limitations of dye-based chromoendoscopy, dye-less chromoendoscopy techniques have been developed. These are subdivided into optical chromoendoscopy techniques, including narrow band imaging (NBI) (Olympus, Tokyo, Japan) and compound band imaging (CBI) (Aohua Photoelectricity, Shanghai, China), and virtual chromoendoscopy techniques, including Fujifilm Intelligent Color Enhancement (FICE) (Fujifilm, Tokyo, Japan) and i-scan (Pentax, 


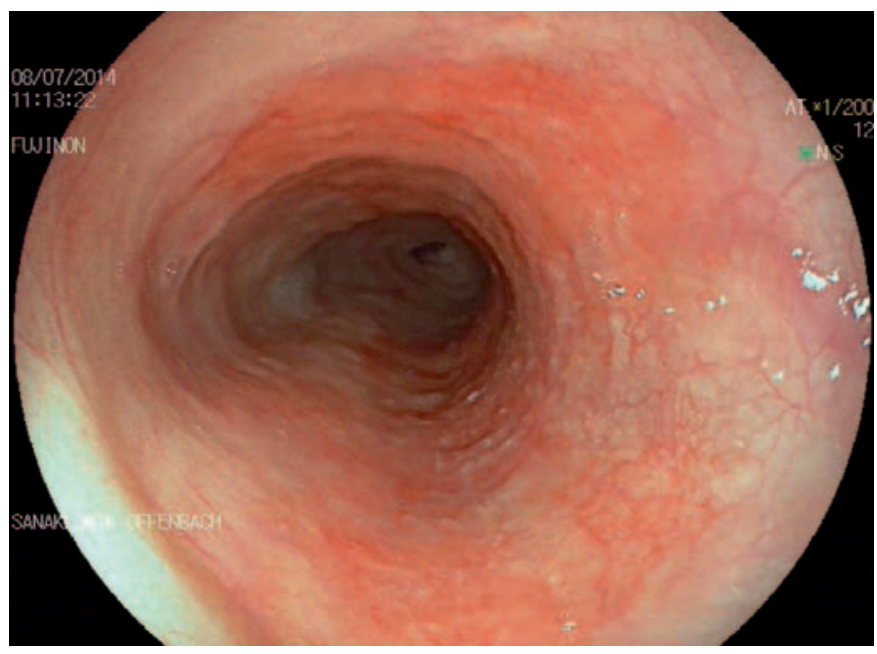

Fig. 3. Early squamous cell carcinoma of the oesophagus. The vanishing mucosal vessels at the 12 o'clock to 3 o'clock position are suspicious for a neoplastic lesion.

Tokyo, Japan). This technique has now been incorporated into all modern video endoscopes and can be applied simply by touching a button on the scope. Optical chromoendoscopy is based on the fact that the depth of penetration of light through tissue depends on its wavelength. Blue light has a shorter wavelength than red light and is reflected in the upper mucosal tissue, allowing visualization of the superficial structures in particular. This digital enhancement can be achieved by using optical filters. In contrast, virtual chromoendoscopy employs digital post-processing to highlight different surface structures [16]. Advanced imaging technologies increase the detection rate for dysplastic Barrett's oesophagus by more than $35 \%$. Sensitivity and specificity were found to be high with 96 and 94\%, respectively, for HGIN in Barrett's oesophagus [17]. However, a prospective randomized study, for example, did not confirm any significant differences between virtual and real chromoendoscopy [18].

Lugol's solution can be used for better visualization in cases of suspected early SCC. This iodine-containing solution stains epithelium that has a high level of glycogen content. SCCs usually have less glycogen and therefore appear white after the solution has been applied (fig. 3, 4). Some studies have evaluated the accuracy of NBI in the diagnostics of early SCC [19-21]. Although NBI seems to have an advantage over white light endoscopy, it remains to be proven that it can replace dye-based chromoendoscopy.

\section{Endomicroscopy and New Imaging Techniques}

The existing guidelines recommend untargeted random biopsies for surveillance in patients with non-neoplastic Barrett's oesophagus, and this can lead to a large number of unnecessary samples being taken. The sensitivity for detecting dysplasia is still unsatisfactory, while the procedure is time-consuming and may be cost-intensive. Great efforts are being made to develop a device

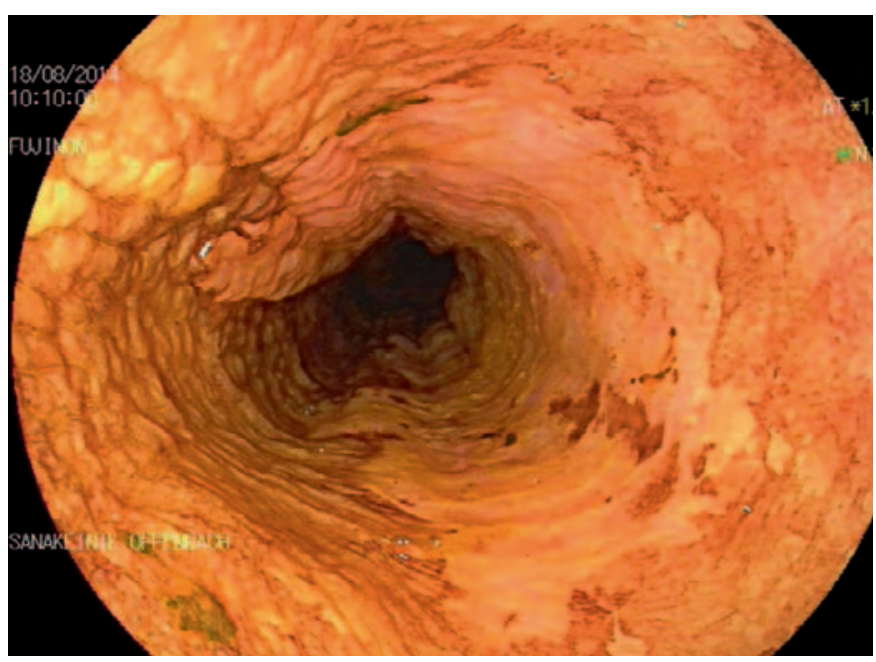

Fig. 4. After staining with Logol's solution the margins are demarcating clearly.

that can be easily used on the oesophageal mucosa to help identify neoplastic areas. A multitude of new imaging techniques have been developed, such as autofluorescence, several spectroscopic techniques, impedance spectroscopy, and polarized light-scattering spectroscopy, to overcome this problem; these are also promising but at an early stage. Confocal laser endomicroscopy (CLE) is a scientifically well-evaluated new method of distinguishing between benign and malignant mucosa in vivo. However, its sensitivity in Barrett's oesophagus varies (68.3-92\%) depending on the examiner's level of experience $[22,23]$. The technique requires advanced skills on the part of the endoscopist to interpret the confocal images, and intravenous injection of fluorescein or topical contrast administration is usually needed $[22,23]$. The overall accuracy of CLE has been reported to be in the range of $81-97 \%$ [23, 24]. However, the method has not become established in routine clinical work so far. Although there is enthusiasm regarding the new techniques now available, the gold standard for diagnosing neoplastic lesions in the oesophagus continues to be histology.

\section{Staging of Early Oesophageal Cancer}

A staging examination should be followed after histologically confirmed oesophageal cancer. Endoscopic ultrasound (EUS) can be used to evaluate the depth of infiltration as well as for lymph node (LN) staging. If there are suspicious LNs (size $>1 \mathrm{~cm}$, round, sharp border, hypoechoic structure) and stage T2 is present (with the tumour extending to the muscularis propria), EUS-guided fineneedle aspiration should be carried out. A positive histological result is an indication for neoadjuvant therapy. If there are multiple suspicious LNs, elastographic endosonography may help identify potentially malignant LNs [25]. Abdominal ultrasound and computed tomography of the thorax complement the staging investigation (fig. 5). 


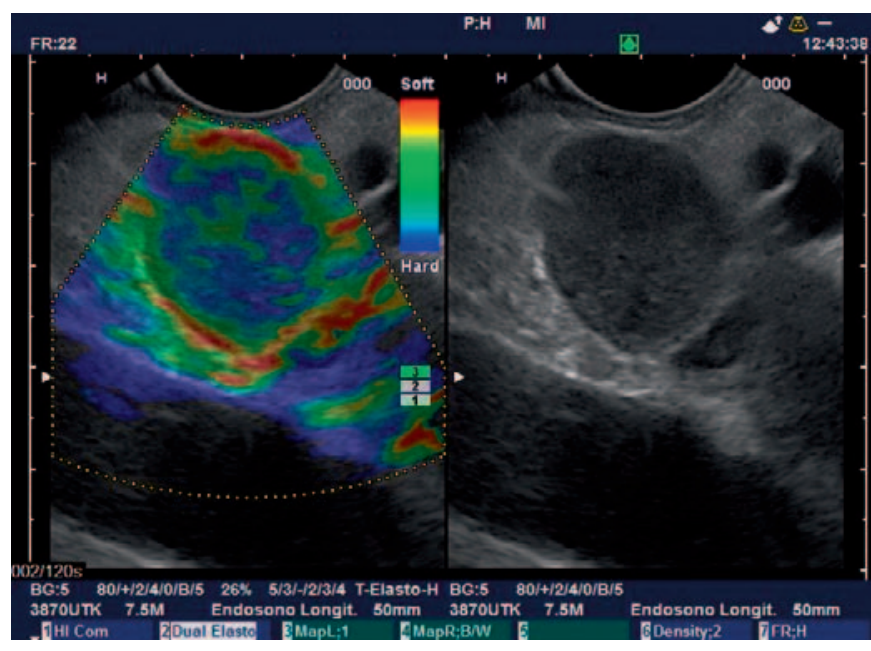

Fig. 5. Endosonographic elastography of a paraoesophageal lymph node which is highly suspicious for malignancy.

\section{Endoscopic Therapy for Early Oesophageal Cancer}

The goal of endoscopic treatment is to achieve complete excision of cancerous lesions from the oesophagus. Endoscopic mucosal resection (EMR) procedures include techniques such as using saline injection followed by cautery snare resection, EMR with a cap fitted onto the endoscope, and endoscopic resection with the use of a band-ligating device. This is carried out by using a cap attached to the tip of the endoscope. After the neoplastic area has been located, the tissue is sucked into the cap and cut with the help of a snare. An alternative method is endoscopic submucosal dissection, which is mainly used for resection in Asian countries. The advantage of the technique is that it allows en bloc resection of the specimen; however, it requires very advanced skills and is extremely time-consuming. The rates of perforation and post-interventional stenosis are higher than with the suck-and-cut technique. In addition, no benefit with regard to the long-term outcome has so far been demonstrated (fig. 6).

Endoscopic resection with the use of a band-ligating device for the treatment of early neoplastic lesions was first described by our own research group in 2000 [26]. The safety of the method was recently confirmed in a study including 1,000 patients. Complete remission was achieved in $94 \%$ of cases, with an overall surveillance period of 5 years. The rate of major complications was $1.5 \%$, and the rate of cancer-associated deaths was $0.2 \%$ [27]. In contrast, oesophageal resection is associated with high rates of perioperative mortality (1-5\%) and morbidity (20-50\%) [28-30]. Current guideline recommendations suggest that every early mucosal AC in the oesophagus should be treated at low-risk stages by using endoscopic resection [31]. Low-risk lesions are defined as pT1m, G1/2, with no lymphatic or vascular invasion and an $\mathrm{R} 0$ resection margin at the base. The same applies to early SCC (pT1m1-m2). If there is multifocal dysplasia or an R1 situation at the lateral margin, patients should undergo a strict surveillance protocol. We suggest gastroscopy at 3-monthly intervals during the first year. If the le-

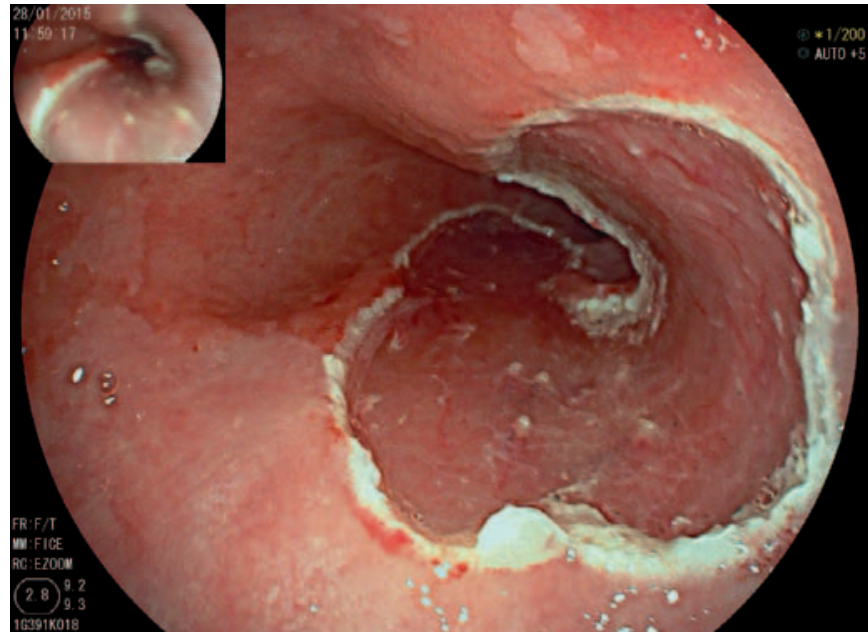

Fig. 6. Wound area after endoscopic resection of an early neoplastic lesion in the distal oesophagus.

sion is unresectable, e.g., if it does not lift as a result of cicatrization, alternative treatment options should be considered.

Although submucosal invasion is considered to be a high-risk situation with a definitive indication for oesophagectomy, there is some evidence that $\mathrm{AC}$ with infiltration into the upper third of the submucosal layer ( $\mathrm{sm} 1)$ may have a good prognosis after endoscopic therapy as well. In a retrospective study, Manner et al. [32] reported complete endoscopic remission in 61 patients with submucosal infiltration. There were no tumour-related deaths in the study group. This treatment option can be considered in patients with high levels of comorbidity and a high surgical risk.

\section{Thermal Ablation}

Thermal ablation is a well-established procedure for residual Barrett's mucosa after treatment for oesophageal AC. It significantly reduces the risk for metachronous dysplasia (by up to 34\%) [33]. Argon plasma coagulation (APC) is usually applied over a probe and is currently considered to be the standard thermal ablation procedure in Germany. Radiofrequency ablation (RFA) as an alternative method has been widely evaluated and is mainly preferred in the USA. In contrast to APC, RFA uses a balloon (or focal catheter) to destroy larger parts of the oesophageal mucosa. Both interventions are associated with a relevant risk for strictures, and buried glands under the newly formed squamous epithelium may occur. A prospective randomized trial comparing APC with RFA has not yet been published. However, RFA is proved as a safe procedure to ablate large parts of intestinal metaplasia [34-36]. Recent studies have also described the use of RFA as a treatment option in mucosa with low-grade and high-grade dysplasia as well as in the treatment of early SCC $[37,38]$. When high-grade dysplasia is diagnosed in a biopsy, there is a potential risk that it represents underestimated AC [39, 40]. In our view, a diagnostic resection is therefore preferable to primary thermal ablation therapy. 
It remains unclear how one should proceed in cases of lowgrade dysplasia. Experts suggest a watch-and-wait strategy, with gastroscopy at 6-monthly intervals. However, there is some evidence that ablation therapy may prevent progression to high-grade dysplasia [41]. The rate of progression without therapy is reported as $1.4-26.5 \%$ per year [10, 42]. Curvers et al. [43] reported that initially diagnosed low-grade intestinal neoplasia was only confirmed after resection in $15 \%$ of cases. We would suggest a diagnostic endoscopic resection in unclear lesions. In addition, all histological samples showing high-grade or low-grade dysplasia should be evaluated by a reference pathologist to confirm the diagnosis [44].

\section{Conclusion}

Although endoscopic treatment in early oesophageal cancer has been established as a safe treatment option with good prognosis and almost no mortality, patients are still being sent to unnecessary surgery. In 2012, we investigated the standard treatment strategies for early oesophageal cancer in eight European countries [45]. 107 university gastroenterology departments were asked to participate in a standardized questionnaire on diagnosis and treatment of early oesophageal cancer. Surprisingly, 37\% would have sent a 50 -year-old patient with multifocal AC to surgery, indicating that the endoscopic treatment option has not been completely accepted. In conclusion, endoscopic therapy should be performed in all patients with early, low-risk cancer. After endoscopic resection of AC all residual Barrett's epithelium has to undergo treatment with thermal ablation to avoid metachronous lesions. When following this advice, endoscopic therapy has an excellent prognosis and can avoid oesophageal resection in most cases.

\section{Disclosure Statement}

The authors have no relevant financial or nonfinancial relationships to disclose.

\section{References}

1 Spechler SJ, Sharma P, Souza RF, Inadomi JM, Shaheen NJ: American Gastroenterological Association technical review on the management of Barrett's esophagus. Gastroenterology 2011;140:e18-52; quiz e13.

2 Rajendra S: Barrett's oesophagus: can meaningful screening and surveillance guidelines be formulated based on new data and rejigging the old paradigm? Best Pract Res Clin Gastroenterol 2015;29:65-75.

3 Ferlay J, Soerjomataram I, Ervik M, Dikshit R, Eser S, Mathers C, Rebelo M, Parkin DM, Forman D, Bray F: GLOBOCAN 2012 v1.0, Cancer Incidence and Mortality Worldwide: IARC CancerBase No. 11. Lyon, International Agency for Research on Cancer; 2013. http:// globocan.iarc.fr (accessed on 31/01/14).

4 Arnold M, Soerjomataram I, Ferlay J, Forman D: Global incidence of oesophageal cancer by histological subtype in 2012. Gut 2015;64:381-387.

5 Chak A, Falk G, Grady WM, et al: Assessment of familiality, obesity, and other risk factors for early age of cancer diagnosis in adenocarcinomas of the esophagus and gastroesophageal junction. Am J Gastroenterol 2009; 104:1913-1921.

6 Hampel H, Abraham NS, El-Serag HB: Meta-analysis: obesity and the risk for gastroesophageal reflux disease and its complications. Ann Intern Med 2005;143:199211.

7 Wu AH, Wan P, Bernstein L: A multiethnic populationbased study of smoking, alcohol and body size and risk of adenocarcinomas of the stomach and esophagus (United States). Cancer Causes Control 2001;12:721-732.

8 Sharma P, Falk GW, Sampliner R, Jon Spechler S, Wang K: Management of nondysplastic Barrett's esophagus: where are we now? Am J Gastroenterol 2009; 104:805-808.

-9 Hvid-Jensen F, Pedersen L, Drewes AM, Sorensen HT, Funch-Jensen P: Incidence of adenocarcinoma among patients with Barrett's esophagus. N Engl J Med 2011; 365:1375-1383.

10 Bhat S, Coleman HG, Yousef F, Johnston BT, McManus DT, Gavin AT, Murray LJ: Risk of malignant progression in Barrett's esophagus patients: results from a large population-based study. J Natl Cancer Inst 2011;103:1049-1057.
Longcroft-Wheaton G, Brown J, Basford P, Cowlishaw D, Higgins B, Bhandari P: Duration of acetowhitening as a novel objective tool for diagnosing high risk neoplasia in Barrett's esophagus: a prospective cohort trial. Endoscopy 2013;45:426-432.

12 Sharma P, Dent J, Armstrong D, et al: The development and validation of an endoscopic grading system for Barrett's esophagus: the Prague C \& M criteria. Gastroenterology 2006;131:1392-1399.

13 Alvarez Herrero L, Curvers WL, van Vilsteren FG, et al: Validation of the Prague C\&M classification of Barrett's esophagus in clinical practice. Endoscopy 2013; 45:876-882.

14 Pohl J, Pech O, May A, Manner H, Fissler-Eckhoff A, Ell C: Incidence of macroscopically occult neoplasias in Barrett's esophagus: are random biopsies dispensable in the era of advanced endoscopic imaging? Am J Gastroenterol 2010;105:2350-2356.

15 Vazquez-Iglesias JL, Alonso-Aguirre P, Diz-Lois MT, Vazquez-Millan MA, Alvarez A, Lorenzo MJ: Acetic acid allows effective selection of areas for obtaining biopsy samples in Barrett's esophagus. Eur J Gastroenterol Hepatol 2007;19:187-193.

16 Neumann H, Fujishiro M, Wilcox CM, Mönkemüller $\mathrm{K}$ : Present and future perspectives of virtual chromoendoscopy with i-scan and optical enhancement technology. Dig Endosc 2014;26(suppl 1):43-51.

17 Mannath J, Subramanian V, Hawkey CJ, Ragunath K: Narrow band imaging for characterization of high grade dysplasia and specialized intestinal metaplasia in Barrett's esophagus: a meta-analysis. Endoscopy 2010; 42:351-359.

18 Pohl J, May A, Rabenstein T, Pech O, Nguyen-Tat M, Fissler-Eckhoff A, Ell C: Comparison of computed virtual chromoendoscopy and conventional chromoendoscopy with acetic acid for detection of neoplasia in Barrett's esophagus. Endoscopy 2007;39:594-598.

19 Lee YC, Wang CP, Chen CC, et al: Transnasal endoscopy with narrow-band imaging and Lugol staining to screen patients with head and neck cancer whose condition limits oral intubation with standard endoscope (with video). Gastrointest Endosc 2009;69:408-417.
20 Takenaka R, Kawahara Y, Okada H, et al: Narrowband imaging provides reliable screening for esophageal malignancy in patients with head and neck cancers. Am J Gastroenterol 2009;104:2942-2948.

21 Ide E, Carneiro FO, Frazao MS, Chaves DM, Sallum RA, de Moura EG, Sakai P, Cecconello I, Maluf-Filho F: Endoscopic detection of early esophageal squamous cell carcinoma in patients with achalasia: narrow-band imaging versus Lugol's staining. J Oncol 2013;2013: 736756.

22 Sharma P, Meining AR, Coron E, et al: Real-time increased detection of neoplastic tissue in Barrett's esophagus with probe-based confocal laser endomicroscopy: final results of an international multicenter, prospective, randomized, controlled trial. Gastrointest Endosc 2011;74:465-472.

23 Kiesslich R, Gossner L, Goetz M, et al: In vivo histology of Barrett's esophagus and associated neoplasia by confocal laser endomicroscopy. Clin Gastroenterol Hepatol 2006;4:979-987.

24 Gaddam S, Mathur SC, Singh M, et al: Novel probebased confocal laser endomicroscopy criteria and interobserver agreement for the detection of dysplasia in Barrett's esophagus. Am J Gastroenterol 2011;106: 1961-1969.

25 Knabe M, Gunter E, Ell C, Pech O: Can EUS elastography improve lymph node staging in esophageal cancer? Surg Endosc 2013;27:1196-1202.

26 Ell C, May A, Gossner L, et al: Endoscopic mucosal resection of early cancer and high-grade dysplasia in Barrett's esophagus. Gastroenterology 2000;118:670-677.

27 Pech O, May A, Manner H, et al: Long-term efficacy and safety of endoscopic resection for patients with mucosal adenocarcinoma of the esophagus. Gastroenterology 2014;146:652-660.e651.

28 Stein HJ, von Rahden BH, Siewert JR: Survival after oesophagectomy for cancer of the oesophagus. Langenbecks Arch Surg 2005;390:280-285.

29 Hölscher AH, Bollschweiler E, Schneider PM, Siewert JR: Early adenocarcinoma in Barrett's oesophagus. Br J Surg 1997;84:1470-1473. 
30 Pech O, Bollschweiler E, Manner H, Leers J, Ell C, Hölscher AH: Comparison between endoscopic and surgical resection of mucosal esophageal adenocarcinoma in Barrett's esophagus at two high-volume centers. Ann Surg 2011;254:67-72.

31 Fitzgerald RC, di Pietro M, Ragunath K, et al: British Society of Gastroenterology guidelines on the diagnosis and management of Barrett's oesophagus. Gut 2014;63:7-42.

-32 Manner H, Pech O, Heldmann Y, May A, Pohl J, Behrens A, Gossner L, Stolte M, Vieth M, Ell C: Efficacy, safety, and long-term results of endoscopic treatment for early stage adenocarcinoma of the esophagus with low-risk sm1 invasion. Clin Gastroenterol Hepatol 2013;11:630-635; quiz e645.

33 Manner H, Rabenstein T, Pech O, Braun K, May A, Pohl J, Behrens A, Vieth M, Ell C: Ablation of residual Barrett's epithelium after endoscopic resection: a randomized long-term follow-up study of argon plasma coagulation vs. surveillance (APE study). Endoscopy 2014;46:6-12.

34 Bulsiewicz WJ, Kim HP, Dellon ES, et al: Safety and efficacy of endoscopic mucosal therapy with radiofrequency ablation for patients with neoplastic Barrett's esophagus. Clin Gastroenterol Hepatol 2013;11:636-642.
5 Gupta M, Iyer PG, Lutzke L, et al: Recurrence of esophageal intestinal metaplasia after endoscopic mucosal resection and radiofrequency ablation of Barrett's esophagus: results from a US Multicenter Consortium. Gastroenterology 2013;145:79-86.e71.

36 Haidry RJ, Dunn JM, Butt MA, et al: Radiofrequency ablation and endoscopic mucosal resection for dysplastic Barrett's esophagus and early esophageal adenocarcinoma: outcomes of the UK National Halo RFA Registry. Gastroenterology 2013;145:87-95.

37 Haidry RJ, Butt MA, Dunn J, et al: Radiofrequency ablation for early oesophageal squamous neoplasia: outcomes form United Kingdom registry. World J Gastroenterol 2013;19:6011-6019.

38 Shaheen NJ, Sharma P, Overholt BF, et al: Radiofrequency ablation in Barrett's esophagus with dysplasia. N Engl J Med 2009;360:2277-2288.

39 Cameron AJ, Carpenter HA: Barrett's esophagus, highgrade dysplasia, and early adenocarcinoma: a pathological study. Am J Gastroenterol 1997;92:586-591.

40 Falk GW, Rice TW, Goldblum JR, Richter JE: Jumbo biopsy forceps protocol still misses unsuspected cancer in Barrett's esophagus with high-grade dysplasia. Gastrointest Endosc 1999;49:170-176.
41 Phoa KN, van Vilsteren FG, Weusten BL, et al: Radiofrequency ablation vs endoscopic surveillance for patients with Barrett esophagus and low-grade dysplasia: a randomized clinical trial. JAMA 2014;311:1209-1217.

42 Wani S, Falk GW, Post J, et al: Risk factors for progression of low-grade dysplasia in patients with Barrett's esophagus. Gastroenterology 2011;141:1179-1186, 1186.e1171.

43 Curvers WL, ten Kate FJ, Krishnadath KK, et al: Lowgrade dysplasia in Barrett's esophagus: overdiagnosed and underestimated. Am J Gastroenterol 2010;105: 1523-1530.

44 Duits LC, Phoa KN, Curvers WL, et al: Barrett's oesophagus patients with low-grade dysplasia can be accurately risk-stratified after histological review by an expert pathology panel. Gut 2015;64:700-706.

45 Heuberger D, Manner H, Ell C, Pech O: How is early Barrett's cancer currently diagnosed and treated in Western Europe? Results of a survey at 52 university hospitals in eight Western European countries. Z Gastroenterol 2012;50:670-676. 